\title{
Impactos de la accesibilidad mediante transporte público en los valores inmobiliarios: una comparación entre las ciudades de Roma y Santander
}

\author{
Rubén Cordera \\ Investigador, Universidad de Cantabria, España \\ Pierluigi Coppola \\ Profesor, Universidad "Tor Vergata” de Roma, Italia \\ Luigi dell'Olio \\ Profesor, Universidad de Cantabria, España \\ Ángel Ibeas \\ Profesor, Universidad de Cantabria, España
}

\section{RESUMEN}

Existe evidencia empírica que avala la hipótesis de que incrementos en la accesibilidad a oportunidades pueden impactar positivamente en los valores inmobiliarios. Sin embargo, esta capitalización de los beneficios de la accesibilidad puede ser desigual en distintas áreas de estudio. En esta investigación se estiman modelos hedónicos y modelos hedónicos espaciales en dos áreas urbanas para comparar si existen diferencias en los impactos de la accesibilidad utilizando transporte público. Las dos áreas de estudio seleccionadas han sido una ciudad media sin grandes problemas de movilidad (Santander, España) y una gran ciudad con elevados problemas de congestión (Roma, Italia). Los modelos hedónicos estimados han considerado la posible existencia de efectos espaciales, habitualmente presentes en los datos inmobiliarios, que pueden generar dependencia en los residuos de los modelos hedónicos. La accesibilidad se ha medido utilizando dos tipos de indicadores: de accesibilidad relativa y gravitatoria. Los resultados permiten afirmar que la accesibilidad fue un factor positivo en el precio de los valores inmobiliarios de ambas ciudades, si bien en el caso de Santander únicamente de forma relativa al centro urbano. Estos resultados son relevantes de cara a implementar políticas de captura del valor que permitan financiar nuevos proyectos de transporte público.

\section{INTRODUCCIÓN}

Los beneficios generados por el transporte en términos de accesibilidad a distintas oportunidades, pueden ser capitalizados por los propietarios inmobiliarios (Martínez, 2000). Los inmuebles son bienes heterogéneos cuyo precio de mercado está influido no sólo por sus características estructurales sino también por las características físicas del entorno y por 
la oferta de transporte disponible. Según el teorema de Henry George, bajo ciertas condiciones teóricas, el gasto agregado en bienes y servicios públicos incrementará las rentas inmobiliarias en el mismo valor (Arnott y Stiglitz, 1979). Por lo tanto, los beneficios generados por equipamientos o infraestructuras de transporte pueden ser capitalizados por los propietarios de los bienes inmobiliarios, abriendo el camino a políticas de captura del valor de cara a financiar el transporte público.

Actualmente ya se están aplicando políticas de captura del valor en distintas ciudades utilizando varios mecanismos como el desarrollo inmobiliario coordinado con el transporte público, la aplicación de impuestos directos a propietarios beneficiados por infraestructuras de transporte o los impuestos a nuevos desarrollos inmobiliarios (Fogarty y America, 2008). En general estas políticas han demostrado ser efectivas de cara a mejorar la sostenibilidad económica y la financiación del transporte público.

El peso de los distintos factores que influyen en el precio de los inmuebles puede estimarse a partir de la técnica conocida como regresión hedónica. Esta técnica fue formalizada por Rosen (1974), si bien ya se había desarrollado previamente a nivel empírico en múltiples casos estudios desde Court (1939). Los modelos de regresión hedónica han resultado ser útiles para evaluar el peso de distintos factores en bienes heterogéneos como los bienes inmobiliarios (Malpezzi, 2008). Estableciendo una regresión de todos los atributos relevantes en el precio del bien, puede estimarse la contribución de cada factor y la disposición a pagar por aumentar o reducir distintas características ambientales. Sin embargo, en este tipo de modelos pueden darse situaciones de autocorrelación espacial en los residuos derivados de distintos efectos que pueden estar presentes en el mercado inmobiliario, produciéndose estimaciones sesgadas o ineficientes de los parámetros.

Este artículo propone la estimación de modelos de regresión hedónica y de modelos de regresión hedónica considerando dependencia espacial entre observaciones con datos agregados obtenidos de las áreas urbanas de Roma (Italia) y Santander (España). Estos modelos permitirán estimar si la accesibilidad mediante transporte público es un factor significativo en los valores inmobiliarios. Los modelos hedónicos serán comparados con los modelos hedónicos espaciales para determinar si éstos se ajustan significativamente mejor a los datos. Los resultados muestran cómo la accesibilidad mediante transporte público es un factor que puede impactar positivamente en los valores inmobiliarios en el caso de Roma, mientras que en Santander únicamente pudo captarse una relación significativa al medir la accesibilidad como el tiempo de viaje al centro urbano. Además mientras que los datos inmobiliarios de Roma presentaron una correlación fuerte en los residuos de los modelos según el índice I de Moran, en el caso de Santander esta correlación fue débil y los modelos hedónicos espaciales no presentaron una mejora significativa respecto a los modelos hedónicos tradicionales.

En la siguiente sección se presenta una breve revisión del estado del arte en el campo de la 
relación entre los valores inmobiliarios y la accesibilidad. La metodología empleada en esta investigación se resume en la sección 3 centrándose en la regresión lineal y la regresión considerando dependencia espacial entre observaciones. En la sección 4 se describen las áreas de estudio y se presentan y discuten los resultados obtenidos en los modelos. Finalmente, las conclusiones alcanzadas en la investigación son resumidas en la sección 5.

\section{ESTADO DEL ARTE SOBRE EL IMPACTO DE LA ACCESIBILIDAD EN LOS VALORES INMOBILIARIOS}

Los modelos hedónicos se han aplicado en múltiples casos de estudio de cara a evaluar los impactos de distintas características ambientales en los precios inmobiliarios. Boyle y Kiel (2001) resumen múltiples casos de estudio centrados en externalidades ambientales, mientras que Debrezion et al. (2007) realizaron un meta-análisis con más de 50 estudios sobre los impactos de las estaciones ferroviarias en los valores inmobiliarios residenciales y comerciales circundantes.

En general los estudios empíricos centrados en los impactos del transporte en los bienes inmobiliarios han mostrado resultados positivos donde los incrementos de accesibilidad implican aumentos en los precios inmobiliarios. Sin embargo distintos estudios también han encontrado efectos neutros (Senior, 2009) e incluso negativos en algunos casos (Bowes y Ihlanfeldt, 2001), especialmente en el ámbito de la accesibilidad a estaciones ferroviarias. Ibeas et al. (2012) encontraron evidencia, a partir de datos desagregados, de la existencia de impactos positivos especialmente si la accesibilidad era medida como el tiempo de viaje al centro urbano, en el área urbana de Santander (España). Wang et al. (2013) confirmaron la importancia de la accesibilidad al transporte en los precios del suelo en el área de estudio de Travis County (EE.UU.) utilizando distintos indicadores como la distancia al centro comercial y de negocios del área urbana o la distancia a carreteras arteriales. Dubé et al. (2013) estimaron modelos para medir la influencia del transporte público en tren en Montreal (Canadá). Los autores pudieron determinar que las viviendas cercanas a las nuevas estaciones experimentaron un incremento en el precio de mercado medio del 2,6\%, un valor que fue capturado a través del incremento de los impuestos municipales generando una recaudación extra de varios millones de dólares.

Otros estudios han realizado estimaciones de los impactos en los valores inmobiliarios derivados de la apertura de nuevos sistemas de Bus Rapid Transit (BRT). Munoz-Raskin (2010) utilizando datos de la ciudad de Bogotá (Colombia) encontraron que las viviendas más próximas a las paradas de bus presentaron un valor casi un $10 \%$ superior a las viviendas situadas a más de 5 minutos. Cervero y Kang (2011) estudiaron el impacto del nuevo sistema BRT de Seúl (Corea del Sur) encontrando incrementos en los valores inmobiliarios de las viviendas a menos de 300 metros de las paradas de bus. Estos incrementos fueron cercanos al $10 \%$. 
Por lo tanto puede decirse que la mayor parte de los estudios realizados han encontrado impactos positivos, si bien diversos dependiendo del área de estudio, de la accesibilidad al transporte público en los valores inmobiliarios. En los apartados siguientes se estimará si estos impactos existen en las ciudades de Santander y Roma y si presentan una magnitud similar en ambos casos.

\section{METODOLOGÍA}

La metodología más utilizada para estimar los impactos de distintos factores ambientales en los bienes inmobiliarios es la regresión hedónica. Este método se basa en un modelo de regresión lineal especificado como:

$$
y=X \beta+\varepsilon
$$

Donde y es el precio o el precio ofertado de una vivienda o de un grupo de viviendas, normalmente especificado en forma logarítmica, $X$ es una matriz con información sobre las variables independientes, tales como características estructurales de las viviendas, variables relacionadas con el transporte y el ambiente etc, $\beta$ es un vector de parámetros a estimar y $\varepsilon$ es un vector de errores independiente e idénticamente distribuidos (IID). En este estudio, las variables de interés contenidas en la matriz X serán los indicadores de accesibilidad, mientras que el resto de variables actuaran como variables de control.

Una introducción a los principales modelos econométricos espaciales desarrollados en la literatura puede encontrarse en LeSage y Pace (2009). El modelo econométrico espacial más conocido para tratar el problema de la presencia de autocorrelación de los residuos en una regresión lineal es el simultaneo autoregresivo (SAR). El modelo SAR asume la existencia de un proceso de dependencia en la variable dependiente. Este modelo se especifica como:

$$
y=\rho W y+X \beta+\varepsilon
$$

Donde $\rho$ es el parámetro de autocorrelación espacial, $\mathrm{W}$ es una matriz $\mathrm{N}$ x $\mathrm{N}$ de pesos espaciales y $\mathrm{N}$ el número de observaciones. El resto de variables son idénticas a las presentes en (1).

La matriz de vecindad $\mathrm{W}$ puede especificarse de múltiples maneras teniendo en cuenta además el tipo de datos disponibles: zonales o puntuales. Los 4 tipos de vecindad más comunes son: tipo reina, tipo torre, número predeterminado de vecinos próximos y distancia de vecindad máxima. La contigüidad tipo reina considera como vecinas aquellas localizaciones adyacentes que comparten un borde o un vértice con la localización dada, mientras que la contigüidad tipo torre considera como vecinas aquellas observaciones que comparten un borde con la localización de referencia (Anselin, 1988). Lesage y Pace (2010a) estudiaron la correlación existente en distintas matrices de vecindad mostrando como la 
influencia de la especificación de $\mathrm{W}$ en las estimaciones de los parámetros es mínima si se interpretan correctamente a partir de las verdaderas derivadas parciales (impactos directos + impactos indirectos), si el número de vecinos próximos es similar y si el modelo está bien especificado.

En el ámbito de los modelos de regresión hedónica aplicados al mercado inmobiliario, la correlación espacial en los residuos de los modelos puede darse por distintos fenómenos. En primer lugar por efecto de difusión en los precios de los inmuebles a observaciones cercanas. En segundo lugar, por la ausencia de equilibrio entre la oferta y la demanda de viviendas en distintas áreas. Por último, la omisión o la medición de forma parcial de alguna variable relevante con efectos diferenciales espacialmente también podría generar un problema de la autocorrelación espacial en los residuos (Bitter et al., 2007). Los modelos econométricos espaciales ayudan a minimizar estos efectos tratando explícitamente la dependencia espacial entre observaciones o entre los residuos de las observaciones.

El concepto de accesibilidad a oportunidades puede medirse utilizando distintos indicadores. Handy y Niemeier (1997) proporcionan una clasificación en tres grandes tipos de indicadores: de oportunidades acumuladas, gravitatorios y basados en la utilidad. Los indicadores más comúnmente empleados son de tipo gravitatorio y tienen la siguiente forma general:

$$
A_{i}=\sum_{j} f\left(E_{j}, C_{i j}\right)
$$

donde $\mathrm{E}_{\mathrm{j}}$ es una medida de atracción de la zona j y $\mathrm{C}_{\mathrm{ij}}$ es una medida del coste de viaje entre las zonas i y j. Los indicadores de oportunidades acumuladas pueden interpretarse como una forma específica de (3) donde $\mathrm{C}_{\mathrm{ij}}$ es igual a 1 si las oportunidades se encuentran dentro de un punto de corte de coste determinado por el analista y 0 en caso contrario (Koenig, 1980). Los indicadores gravitatorios presentan por lo tanto la ventaja frente a los indicadores de oportunidades acumuladas de ponderar de forma diferenciada las oportunidades según los costes de viaje a las mismas sin establecer un punto de corte binario. Además los indicadores gravitatorios son de tipo zonal a diferencia de los indicadores basados en la utilidad que son de tipo individual con lo que proporcionan una mejor comprensión de la relación entre el transporte y los usos del suelo (Horner, 2004).

En este trabajo se ha optado por emplear los indicadores de tipo gravitatorio propuestos por Cascetta (2009) y Coppola y Nuzzolo (2011). Estos autores diferencian entre la accesibilidad activa de una zona que puede definirse como la capacidad para alcanzar las oportunidades presentes en otras zonas, y la accesibilidad pasiva que se define como la capacidad de una zona para ser alcanzada desde otras zonas. Formalmente los indicadores se formulan como:

$$
\left.A C C_{-} A C T_{i}=\sum_{j}\left[\exp \left(\alpha_{2} \cdot C_{i j}\right)\right) \cdot E_{j}^{\alpha_{1}}\right]
$$




$$
\left.A C C_{-} P A S_{j}=\sum_{i}\left[\exp \left(\alpha_{4} \cdot C_{i j}\right)\right) \cdot P_{i}^{\alpha 3}\right]
$$

Donde $\mathrm{P}_{\mathrm{i}}$ es la población o el número de hogares presentes en la zona $\mathrm{i}, \mathrm{E}_{\mathrm{j}} \mathrm{y}_{\mathrm{ij}}$ son iguales a las presentes en la ecuación (3) y $\alpha_{1}, \alpha_{2}, \alpha_{3}$ y $\alpha_{4}$ son parámetros a estimar. Estos indicadores se pueden calibrar fácilmente mediante mínimos cuadrados ordinarios (OLS) transformando ambos lados de las expresiones (4) y (5) de forma logarítmica. Además en áreas con un marcado carácter monocéntrico, el tiempo de viaje al centro urbano puede ser un buen indicador de accesibilidad relativa a las oportunidades de empleo, de ocio, de comercio y de otros motivos de viaje. En áreas monocéntricas, la correlación entre los indicadores gravitatorios y los indicadores de accesibilidad relativa al centro urbano puede ser por lo tanto elevada.

\section{4. ÁREAS DE ESTUDIO Y RESULTADOS}

\subsection{Datos disponibles y áreas de estudio}

Para la estimación de los modelos de regresión hedónica se ha contado con dos bases de datos para las ciudades y las áreas de influencia de Roma y Santander. En ambos casos, los datos han estado disponibles en forma agregada con un total de 211 zonas para el caso de Roma y de 42 zonas para el caso de Santander. Las áreas de estudio tienen características claramente diferenciadas. Roma y su área de influencia, presentan casi dos millones y medio de habitantes y un millón de empleos. El área tiene un centro principal formado por los barrios históricos (Municipio I) y dos centros secundarios en el área de negocios EUR y en el puerto de Ostia. En total estas tres áreas agrupan aproximadamente el $20 \%$ del empleo de la zona de estudio. La ciudad presenta además problemas de congestión notables. Santander en cambio es una ciudad media con un área de influencia formada por 8 municipios presentando en total 260.000 habitantes y algo más de 100.000 empleos. El área presenta un marcado carácter monocéntrico con el núcleo principal de Santander concentrando el 20\% de los empleos totales. Actualmente la ciudad tiene problemas de congestión moderados especialmente en los accesos a la ciudad.

En la Figura 1 se muestran los precios medios (en euros por metro cuadrado) en cada una de las zonas. En el caso de Roma, los precios siguen un patrón marcadamente concéntrico desde el centro histórico de la ciudad hacia la periferia con una caída cerca de la circunvalación principal de la ciudad (A-90). En Santander los precios siguen más bien un gradiente norte - sur, desde la costa al interior, y en el caso del núcleo urbano de Santander de este a oeste.

Las variables contenidas en la base de datos para ambas ciudades pueden consultarse en la Tabla 2 y la Tabla 3. Los precios por metro cuadrado (variable P) fueron claramente más elevados en Roma que en Santander con una medida de 3440 euros versus 2661 euros. Además el rango de precios en Roma fue también más amplio con un máximo de 8250 
euros $/ \mathrm{m}^{2}$. Las variables POP, HOUSE, SQM y EMP miden la población, el número de viviendas, los metros cuadrados residenciales y el número de empleos presentes en cada zona respectivamente.

Los indicadores de accesibilidad activa y pasiva (ACCA y ACCP) mediante transporte público, se especificaron con los empleos y la población presente en las zonas respectivamente. En ambos casos los indicadores tuvieron un menor valor en Santander derivado del menor número de empleos y población de las zonas. Los parámetros calibrados para los indicadores pueden consultarse en la Tabla 1.

\begin{tabular}{ccc}
\hline Parámetro & Roma & Santander \\
\hline$\alpha_{1}$ & 0,05 & 0,02 \\
$\alpha_{2}$ & $-0,1$ & $-0,1$ \\
$\alpha_{3}$ & 0,85 & 0,02 \\
$\alpha_{4}$ & 1,22 & $-0,1$ \\
\hline
\end{tabular}

Tabla 1. Parámetros calibrados para los indicadores de accesibilidad

El tiempo de viaje al centro urbano en minutos utilizando transporte público (CBD) se especificó en el caso de Roma considerando únicamente como centro el área de los barrios históricos y en Santander el centro de la ciudad. El tiempo de viaje en transporte público no sólo incluye el tiempo a bordo del vehículo, sino también los tiempos de espera y de transbordo en su caso para cada zona. En Roma el transporte público considerado estuvo formado por el bus y el metro, mientras que en Santander estuvo formado por el sistema de autobús urbano e interurbano. El indicador fue nuevamente muy superior en el caso de Roma, con un valor medio superior a los 67 minutos frente a 49 minutos para el caso de Santander. En cuanto a la variable de cobertura espacial en transporte público (TRANS) ésta fue más elevada en el caso de Santander con una media del $73 \%$ de la zona cubierta, si bien hay que considerar que el área de estudio de Santander es considerablemente más reducida que el área de Roma (299 $\mathrm{Km}^{2}$ en el caso de Santander y $1326 \mathrm{Km}^{2}$ en el caso de Roma). En ambos casos la cobertura se midió a través de un buffer en las paradas de transporte público de 400 metros (máximo de 6 minutos de tiempo de acceso considerando una velocidad de $4 \mathrm{~km} / \mathrm{h})$.

El resto de variables son de tipo dummy tomando un valor igual a 1 si la zona pertenece al centro urbano (CEN), si forma parte de un área comercial (COM), si presenta una zona verde (GREEN) y si pertenece a un área de especial prestigio (PG), una característica más subjetiva a determinar por el analista. En el caso de las variables dummy, destaca el hecho de que la proporción de zonas presentando cada una de las características es muy similar en ambas áreas urbanas, si bien las zonas pertenecientes al centro y sobre todo a un área comercial son más numerosas en el caso de Roma que en Santander. 

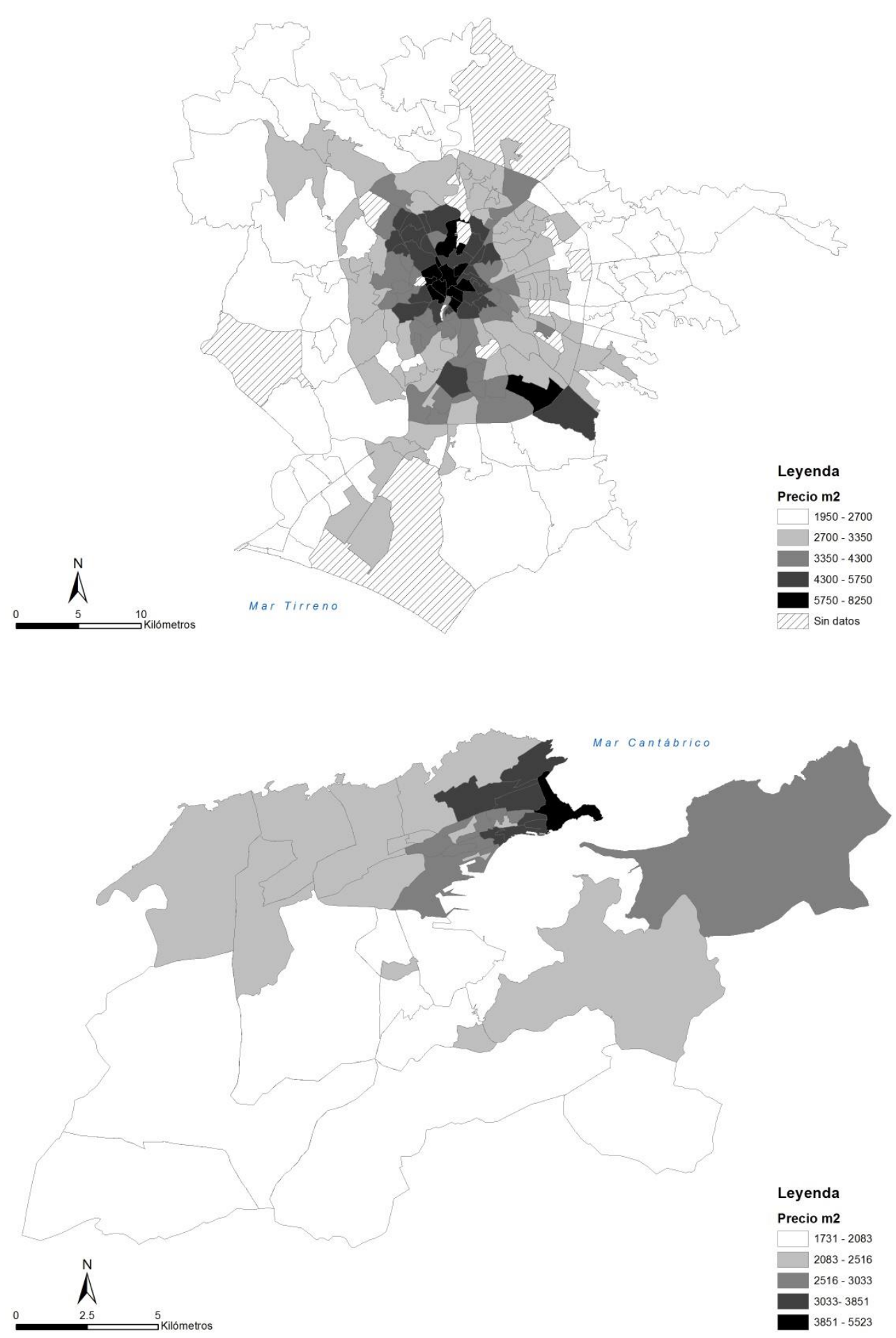

Figura 1- Precios inmobiliarios (euros $/ \mathrm{m}^{2}$ ) en las ciudades de Roma (arriba) y Santander (abajo)

\begin{tabular}{llllll}
\hline Variable Descripción & Unidades & Media & $\begin{array}{c}\text { Desviación } \\
\text { Estándar }\end{array}$ & Mínimo & Máximo \\
\hline
\end{tabular}




\begin{tabular}{|c|c|c|c|c|c|c|}
\hline $\mathrm{P}$ & $\begin{array}{c}\text { Precio de los } \\
\text { inmuebles por } \mathrm{m}^{2}\end{array}$ & Euros & 3440,40 & 1208,81 & 1950 & 8250 \\
\hline POP & Población & - & 11790,50 & 9738,86 & 274 & 49693 \\
\hline HOUSE & Viviendas & - & 5424,67 & 5082,74 & 89 & 23969 \\
\hline SMQ & $\begin{array}{l}\text { Metros Cuadrados } \\
\quad \text { construidos }\end{array}$ & $\mathrm{m}^{2}$ & 457504,1 & 404101,4 & 4989 & 1914407 \\
\hline EMP & Empleos & - & 5584,83 & 6160,20 & 159 & 36794 \\
\hline ACCA & $\begin{array}{l}\text { Accesibilidad } \\
\text { Activa mediante } \\
\text { transporte público } \\
\text { Accesibilidad }\end{array}$ & - & 4,34 & 4,74 & 0 & 23,48 \\
\hline $\mathrm{ACCP}$ & $\begin{array}{l}\text { Pasiva mediante } \\
\text { transporte público }\end{array}$ & - & 4,44 & 4,68 & 0 & 21,43 \\
\hline CBD & $\begin{array}{l}\text { Tiempo de viaje al } \\
\text { centro en transporte } \\
\text { público }\end{array}$ & Minutos & 67,04 & 28,69 & 13,26 & 166,10 \\
\hline TRANS & $\begin{array}{l}\text { Cobertura de } \\
\text { transporte público } \\
\text { en la zona }\end{array}$ & $0-1$ & 0,19 & 0,26 & 0 & 1 \\
\hline CEN & $\begin{array}{l}\text { Pertenencia a la } \\
\text { zona centro }\end{array}$ & $1 / 0$ & 0,07 & 0,25 & 0 & 1 \\
\hline $\mathrm{COM}$ & $\begin{array}{l}\text { Pertenencia a una } \\
\text { zona comercial }\end{array}$ & $1 / 0$ & 0,06 & 0,20 & 0 & 1 \\
\hline GREEN & $\begin{array}{l}\text { Presencia de zona } \\
\text { verde }\end{array}$ & $1 / 0$ & 0,14 & 0,33 & 0 & 1 \\
\hline PG & $\begin{array}{c}\text { Zona de especial } \\
\text { prestigio }\end{array}$ & $1 / 0$ & 0,12 & 0,31 & 0 & 1 \\
\hline
\end{tabular}

Tabla 2 - Estadísticos descriptivos de las variables contenidas en la base de datos de Roma (N=211 zonas)

\begin{tabular}{|c|c|c|c|c|c|c|}
\hline Variable & Descripción & Unidades & Media & $\begin{array}{l}\text { Desviación } \\
\text { Estándar }\end{array}$ & Mínimo & Máximo \\
\hline $\mathrm{P}$ & $\begin{array}{c}\text { Precio de los } \\
\text { inmuebles por } \mathrm{m}^{2}\end{array}$ & Euros & 2661,31 & 716,31 & 1731 & 5523 \\
\hline POP & Población & - & 6683,76 & 1379,54 & 4310 & 9849 \\
\hline HOUSE & Viviendas & - & 2095 & 718,95 & 987 & 3804 \\
\hline SMQ & $\begin{array}{l}\text { Metros Cuadrados } \\
\quad \text { construidos }\end{array}$ & $\mathrm{m}^{2}$ & 182608,7 & 56756,90 & 94266 & 321264 \\
\hline EMP & Empleos & - & 2388,95 & 2406,61 & 441 & 11359 \\
\hline ACCA & $\begin{array}{l}\text { Accesibilidad } \\
\text { Activa mediante } \\
\text { transporte público } \\
\text { Accesibilidad }\end{array}$ & - & 0,97 & 0,92 & 0,04 & 3,56 \\
\hline $\mathrm{ACCP}$ & $\begin{array}{l}\text { Pasiva mediante } \\
\text { transporte público }\end{array}$ & - & 0,99 & 0,95 & 0 & 3,36 \\
\hline CBD & $\begin{array}{l}\text { Tiempo de viaje al } \\
\text { centro en transporte } \\
\text { público }\end{array}$ & Minutos & 49,31 & 20,69 & 13,45 & 82,24 \\
\hline TRANS & $\begin{array}{l}\text { Cobertura de } \\
\text { transporte público } \\
\text { en la zona }\end{array}$ & $0-1$ & 0,73 & 0,33 & 0,08 & 1 \\
\hline
\end{tabular}




\begin{tabular}{ccccccc} 
CEN & $\begin{array}{c}\text { Pertenencia a la } \\
\text { zona centro }\end{array}$ & $1 / 0$ & 0,05 & 0,22 & 0 & 1 \\
COM & $\begin{array}{c}\text { Pertenencia a una } \\
\text { zona comercial }\end{array}$ & $1 / 0$ & 0,12 & 0,33 & 0 & 1 \\
GREEN & $\begin{array}{c}\text { Presencia de zona } \\
\text { verde }\end{array}$ & $1 / 0$ & 0,14 & 0,35 & 0 & 1 \\
PG & $\begin{array}{c}\text { Zona de especial } \\
\text { prestigio }\end{array}$ & $1 / 0$ & 0,10 & 0,30 & 0 & 1 \\
\hline Tabla 3 - Estadísticos descriptivos de las variables contenidas en la base de datos de \\
Santander $(\mathbf{N}=\mathbf{4 2}$ zonas)
\end{tabular}

\subsection{Resultados de los modelos}

Los parámetros estimados para los modelos de Roma y Santander se resumen en la Tabla 4 y en la Tabla 5. Los modelos R-1, R-2, S-1 y S-2 corresponden regresiones hedónicas, mientras que los modelos R-3, R-4, S-3 y S-4 son modelos espaciales autoregresivos considerando dependencia espacial en el precio de los inmuebles. La variable precio en todos los casos se ha especificado en forma logarítmica. Esta forma funcional presenta varias ventajas como la reducción de la posible heterocedasticidad en los residuos de los modelos (Malpezzi, 2008). La especificación de la variable dependiente en forma logarítmica permite además interpretar los parámetros estimados como semielasticidades, es decir, como el cambio porcentual de la variable dependiente por un cambio unitario en la variable independiente manteniendo el resto de variables constantes. En el caso de las variables dummy, el efecto del parámetro debe estimarse utilizando la expresión: $\left[\exp \left(\beta_{\mathrm{n}}\right)-1\right] * 100$, donde $\beta_{\mathrm{n}}$ es el parámetro de la variable dummy considerada (Halvorsen y Palmquist, 1980).

En la especificación de los modelos se han excluido variables que presentaban una alta correlación entre si y que podían por lo tanto generar problemas de colinealidad en los modelos. En primer lugar las variables POP, HOUSE y SQM presentaron una correlación superior a 0,6 por lo que se eligió utilizar únicamente la variable POP en los modelos. Además los modelos se especificaron o bien con el indicador de accesibilidad gravitatorio, o bien con el indicador de tiempo de viaje al centro para detectar cual proporcionaba un mejor ajuste a los datos. Ambos indicadores no se utilizaron simultáneamente ya que presentaban una correlación elevada (superior a 0,6). Además ACCA y ACCP también estaban fuertemente correlacionadas en ambas áreas de estudio (índice de correlación superior a 0,8 ) por lo que se optó por utilizar únicamente la accesibilidad activa para evaluar su influencia en los valores inmobiliarios.

Los modelos R-1 y S-1 quedaron especificados finalmente con un total de 8 variables adoptando la siguiente forma funcional: 


$$
\begin{aligned}
& \ln \left(P_{i}\right)=\beta_{1}+\beta_{2} \text { POP }_{i}+\beta_{3} \text { EMP }_{i}+\beta_{4} \text { ACCA }_{i}+\beta_{5} \text { TRANS }_{i}+\beta_{6} \text { CEN }_{i}+\beta_{7} \text { COM }_{i} \\
& +\beta_{8} \text { GREEN }_{i}+\beta_{9} P G_{i}+\varepsilon_{i}
\end{aligned}
$$

El ajuste del modelo R-1 fue de un 74\% de la variancia explicada. La población de la zona (variable POP) tuvo un efecto negativo pequeño con una significatividad cercana a un nivel de confianza del 95\%. Los empleos presentes en la zona sin embargo (EMP) resultaron no ser significativos. Entre las variables dummy que consideran distintas características zonales, únicamente la presencia de zona verde y el prestigio especial del área resultaron ser significativas. Sin embargo, la presencia de zona verde presentó un contraintuitivo signo negativo, mientras que el prestigio fue claramente un factor positivo e importante con un aumento del $40 \%$ en el precio medio de los inmuebles. La variable de accesibilidad gravitatoria resultó ser significativa con un aumento del $4 \%$ en el precio de los inmuebles por unidad adicional en el indicador. La cobertura de transporte público en la zona (TRANS) presentó un signo negativo y no significativo. Este modelo se especificó también con el tiempo de viaje en transporte público al centro urbano histórico (CBD) en lugar del indicador gravitatorio (modelo R-2). En este caso la bondad de ajuste del modelo aumento hasta un 0,77 en el indicador $R^{2}$. La variable número de empleos pasó a ser significativa, mientras que la cobertura de las paradas de transporte público presentó aún más claramente un parámetro no significativamente distinto de cero. Entre las variables dummy considerando características ambientales, la presencia de zona verde cambio de signo pero dejando el parámetro de ser significativo. En general puede decirse por lo tanto, que el indicador de accesibilidad relativa permitió mejorar ligeramente el ajuste y la coherencia de los parámetros respecto al indicador de accesibilidad gravitatorio.

En los residuos de los modelos R-1 y R-2 se aplicó un índice I de Moran de cara a comprobar si existía en grado de correlación espacial significativa. En ambos modelos esta correlación resultó ser positiva y fuerte. De cara a determinar qué modelo espacial podía ser más apto para capturar esta correlación, se aplicó un test robusto de multiplicadores de Lagrange resultando que el test LM - lag fue claramente significativo y con valores muy superiores al test LM - error.

Dados estos resultados, los modelos R-3 y R-4 se especificaron con las mismas variables presentes respectivamente en R-1 y R-2 pero considerando también la existencia de dependencia espacial en el precio de inmuebles vecinos. La matriz de vecindad adoptada estuvo compuesta por las 5 zonas más próximas. Se prefirió seleccionar este tipo de matriz de vecindad frente a otras alternativas, dado que asegura una distribución homogénea de vecindad entre observaciones, algo recomendable ya que el cálculo de las propiedades asintóticas de los estimadores y de los test estadísticos se basan en la hipótesis de existencia de regularidad (Anselin, 2002).

\begin{tabular}{lllll}
\hline Variable & R-1 & R-2 & R-3 & R-4 \\
& & & & \\
\hline
\end{tabular}




\begin{tabular}{|c|c|c|c|c|}
\hline (Intercepto) & $\begin{array}{l}7,921 \\
(, 000)\end{array}$ & $\begin{array}{l}8,478 \\
(, 000)\end{array}$ & $\begin{array}{l}2,498 \\
(, 000)\end{array}$ & $\begin{array}{l}2,999 \\
(, 000)\end{array}$ \\
\hline \multirow[t]{2}{*}{ POP } & $-0,000$ & $-0,000$ & $-0,000$ & $-0,000$ \\
\hline & $(, 063)$ & $(, 008)$ & $(, 089)$ & $(, 037)$ \\
\hline \multirow[t]{2}{*}{ EMP } & 0,000 & 0,000 & 0,000 & 0,000 \\
\hline & $(, 730)$ & $(, 014)$ & $(, 295)$ & $(, 044)$ \\
\hline \multirow[t]{2}{*}{$\mathrm{ACCA}$} & 0,041 & - & 0,013 & - \\
\hline & $(, 000)$ & & $(, 000)$ & \\
\hline \multirow[t]{2}{*}{ CBD } & - & $-0,006$ & - & $-0,002$ \\
\hline & & $(, 000)$ & & $(, 000)$ \\
\hline \multirow[t]{2}{*}{ TRANS } & $-0,094$ & 0,008 & $-0,027$ & $-0,002$ \\
\hline & $(, 115)$ & $(, 873)$ & $(, 524)$ & $(, 938)$ \\
\hline \multirow[t]{2}{*}{ CEN } & 0,072 & 0,145 & 0,040 & 0,069 \\
\hline & $(, 138)$ & $(, 002)$ & $(, 232)$ & $(, 042)$ \\
\hline \multirow[t]{2}{*}{$\mathrm{COM}$} & $-0,033$ & $-0,097$ & $-0,024$ & $-0,046$ \\
\hline & $(, 554)$ & $(, 063)$ & $(, 538)$ & $(, 224)$ \\
\hline \multirow[t]{2}{*}{ GREEN } & $-0,070$ & 0,031 & $-0,054$ & $-0,016$ \\
\hline & $(, 054)$ & $(, 391)$ & $(, 031)$ & $(, 549)$ \\
\hline \multirow[t]{2}{*}{ PG } & 0,338 & 0,314 & 0,165 & 0,162 \\
\hline & $(, 000)$ & $(, 000)$ & $(, 000)$ & $(, 000)$ \\
\hline \multirow[t]{2}{*}{$\rho$} & - & - & 0,683 & 0,645 \\
\hline & & & $(, 000)$ & $(, 000)$ \\
\hline$\overline{\mathrm{R}^{2}}$ & 0,74 & 0,77 & - & - \\
\hline $\mathrm{R}^{2}$ ajustado & 0,73 & 0,76 & - & - \\
\hline Test F & 71,99 & 82,74 & - & - \\
\hline & $(, 000)$ & $(, 000)$ & & \\
\hline Log-verosimilitud & 95,78 & 106,84 & 157,44 & 162,12 \\
\hline AIC & $-173,56$ & $-195,69$ & $-292,88$ & $-302,24$ \\
\hline I de Moran & 0,37 & 0,33 & 0,01 & 0,02 \\
\hline & $(, 000)$ & $(, 000)$ & $(, 581)$ & $(, 511)$ \\
\hline LM - Lag robusto & 42,55 & 43,56 & - & - \\
\hline & $(, 000)$ & $(, 000)$ & & \\
\hline LM - Error robusto & 3,00 & 1,95 & - & - \\
\hline & $(, 083)$ & $(, 162)$ & & \\
\hline LR test & - & - & 123,32 & 110,55 \\
\hline & & & $(, 000)$ & $(, 000)$ \\
\hline
\end{tabular}

Tabla 4 - Modelos estimados para el área de Roma (entre paréntesis los p - valor con la significancia estadística de los parámetros)

Los parámetros estimados en los modelo R-3 y R-4 fueron en general menores a los estimados en R-1 R-2, si bien deben considerarse los impactos totales tal y como se detallará en el apartado siguiente. Aun así tanto el indicador de accesibilidad como el indicador del tiempo de viaje al centro presentaron parámetros significativos con estimaciones de un incremento del $1,3 \%$ en el precio medio de los inmuebles de las zonas por unidad de accesibilidad adicional o de reducción de un $0,2 \%$ por minuto de viaje adicional al centro urbano. La presencia de una mayor cobertura espacial de transporte público siguió sin ser una variable significativa.

Los test de razón de verosimilitud (LR test) entre los modelos SAR y los modelos hedónicos fueron claramente significativos por lo que se puede afirmar que los modelos R-3 y R-4 se 
ajustaron claramente mejor a los datos. Los parámetros $\rho$ estimados fueron en ambos casos claramente significativos y el test I de Moran realizado en los residuos de los modelos espaciales mostró como la correlación en los residuos dejó de estar presente.

\begin{tabular}{|c|c|c|c|c|}
\hline Variable & S-1 & $\mathrm{S}-2$ & S-3 & $\mathrm{S}-4$ \\
\hline$\overline{\text { (Intercepto) }}$ & $\begin{array}{l}7,666 \\
(, 000)\end{array}$ & $\begin{array}{l}8,140 \\
(, 000)\end{array}$ & $\begin{array}{l}5,978 \\
(, 000)\end{array}$ & $\begin{array}{l}6,592 \\
(, 000)\end{array}$ \\
\hline POP & $\begin{array}{c}-0,000 \\
(, 089)\end{array}$ & $\begin{array}{c}-0,000 \\
(, 028)\end{array}$ & $\begin{array}{c}-0,000 \\
(, 170)\end{array}$ & $\begin{array}{r}-0,000 \\
(, 039)\end{array}$ \\
\hline EMP & $\begin{array}{l}0,000 \\
(, 559)\end{array}$ & $\begin{array}{l}0,000 \\
(, 247)\end{array}$ & $\begin{array}{l}0,000 \\
(, 513)\end{array}$ & $\begin{array}{l}0,000 \\
(, 171)\end{array}$ \\
\hline ACCA & $\begin{array}{l}0,045 \\
(, 213)\end{array}$ & - & $\begin{array}{l}0,029 \\
(, 370)\end{array}$ & - \\
\hline CBD & - & $\begin{array}{r}-0,005 \\
(, 008)\end{array}$ & - & $\begin{array}{r}-0,005 \\
(, 003)\end{array}$ \\
\hline TRANS & $\begin{array}{l}0,396 \\
(, 000)\end{array}$ & $\begin{array}{l}0,219 \\
(, 053)\end{array}$ & $\begin{array}{c}0,328 \\
(, 000)\end{array}$ & $\begin{array}{l}0,157 \\
(, 127)\end{array}$ \\
\hline CEN & $\begin{array}{r}-0,277 \\
(, 187)\end{array}$ & $\begin{array}{c}-0,406 \\
(, 046)\end{array}$ & $\begin{array}{c}-0,289 \\
(, 103)\end{array}$ & $\begin{array}{r}-0,423 \\
(, 012)\end{array}$ \\
\hline $\mathrm{COM}$ & $\begin{array}{l}-0,016 \\
(, 844)\end{array}$ & $\begin{array}{l}0,043 \\
(, 607)\end{array}$ & $\begin{array}{l}0,016 \\
(, 832)\end{array}$ & $\begin{array}{l}0,065 \\
(, 354)\end{array}$ \\
\hline GREEN & $\begin{array}{l}0,280 \\
(, 001)\end{array}$ & $\begin{array}{l}0,272 \\
(, 001)\end{array}$ & $\begin{array}{l}0,275 \\
(, 000)\end{array}$ & $\begin{array}{l}0,268 \\
(, 000)\end{array}$ \\
\hline PG & $\begin{array}{l}0,282 \\
(, 033)\end{array}$ & $\begin{array}{l}0,231 \\
(, 057)\end{array}$ & $\begin{array}{l}0,292 \\
(, 008)\end{array}$ & $\begin{array}{l}0,246 \\
(, 017)\end{array}$ \\
\hline$\rho$ & - & - & $\begin{array}{l}0,215 \\
(, 139)\end{array}$ & $\begin{array}{l}0,193 \\
(, 136)\end{array}$ \\
\hline$\overline{\mathrm{R}^{2}}$ & 0,74 & 0,78 & - & - \\
\hline $\mathrm{R}^{2}$ ajustado & 0,68 & 0,73 & - & - \\
\hline Test F & $\begin{array}{l}12,03 \\
(, 000)\end{array}$ & $\begin{array}{l}14,96 \\
(, 000)\end{array}$ & - & - \\
\hline Log-verosimilitud & 29,73 & 33,23 & 30,79 & 34,30 \\
\hline AIC & $-41,46$ & $-48,46$ & $-39,56$ & $-46,60$ \\
\hline I de Moran & $\begin{array}{c}0,07 \\
(, 055)\end{array}$ & $\begin{array}{c}0,01 \\
(, 369)\end{array}$ & $\begin{array}{l}-0,08 \\
(, 481)\end{array}$ & $\begin{array}{l}-0,09 \\
(, 345)\end{array}$ \\
\hline LM - Lag robusto & $\begin{array}{c}9,10 \\
(, 002)\end{array}$ & $\begin{array}{c}6,46 \\
(, 011)\end{array}$ & - & - \\
\hline LM - Error robusto & $\begin{array}{c}1,92 \\
(, 166)\end{array}$ & $\begin{array}{c}2,29 \\
(, 130)\end{array}$ & - & - \\
\hline LR test & - & - & $\begin{array}{c}2,10 \\
(, 147)\end{array}$ & $\begin{array}{c}2,14 \\
(, 144)\end{array}$ \\
\hline
\end{tabular}

Tabla 5- Modelos estimados para el área de Santander (entre paréntesis los $p$ - valor con la significancia estadística de los parámetros)

Los modelos estimados para el caso de Santander se especificaron con las mismas variables que en el caso de Roma así como con la misma matriz de vecindad en el caso de S-3 y S-4. En los modelos para Santander, los parámetros estimados en la variable ACCA, fueron algo superiores a los estimados para los modelos de Roma, si bien en este caso no fueron claramente significativos. Si fueron en cambio significativamente distintos de cero según el test $t$ los parámetros estimados para la distancia al centro urbano (CBD) con un peso de $0,5 \%$ 
de reducción en el precio zonal medio de los inmuebles por minuto adicional de viaje al centro urbano (véase Figura 2 con la comparativa de los efectos parciales para esa variable en los modelos R-2 y S-2). La variable TRANS, a diferencia de lo señalado para Roma, fue más claramente significativa especialmente en los modelos S-1 y S-3 aunque en los modelos especificados con la variable CBD la significatividad se redujo notablemente. Otras variables ambientales que mostraron ser relevantes fueron la presencia de zona verde, con impactos positivos en torno al $27 \%$ de incremento medio en el precio de los inmuebles, y PG también con más de un $25 \%$ de incremento según el modelo.

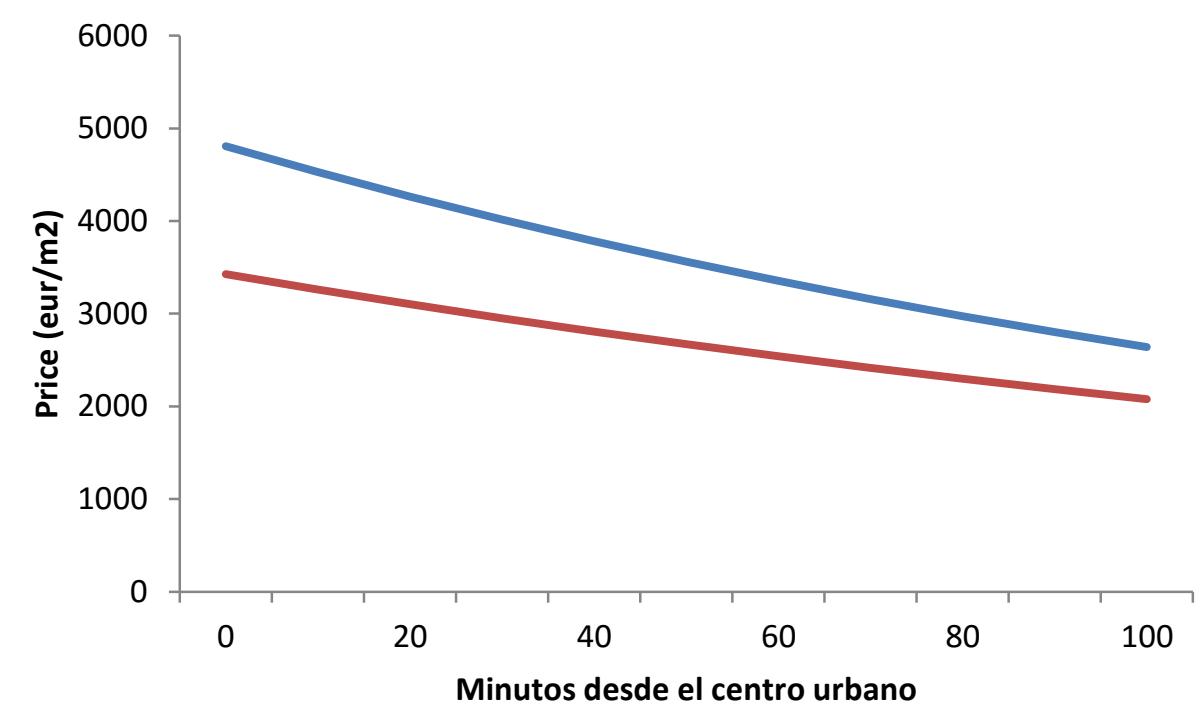

Figura 2- Efecto parcial de la variable CBD en los modelos R-2 (Azul) y S-2 (Rojo)

La bondad de ajuste en los modelos para Santander fue similar según el $\mathrm{R}^{2}$ al caso de Roma. Sin embargo la presencia de correlación espacial en los residuos de los modelos fue muy débil y los test LR presentaron valores muy bajos, lo que implica que los modelos SAR no mejoraron el ajuste respecto a los modelos de regresión hedónica simples.

\subsection{Impactos totales en los modelos espaciales autoregresivos}

De cara a la correcta interpretación de los parámetros de los modelos SAR, se calcularon los impactos totales de las variables, los cuales tienen en cuenta tanto el efecto directo como el efecto indirecto de difusión espacial que provocan las variables independientes (véase Tabla 6) (LeSage y Pace, 2010b). Teniendo en cuenta estos efectos directos e indirectos, puede verse como los modelos SAR permiten inferir que el efecto total del cambio de ciertas variables, incluidas ACCA y CBD, es mayor que el estimado en los modelos hedónicos. Este efecto fue especialmente notable en el caso de los modelos R-3 y R-4, donde la presencia de correlación espacial en los residuos fue más notable. Considerando estos impactos totales, un minuto adicional de tiempo de viaje al centro urbano en transporte público implicó en 
Roma un descenso de $0,7 \%$ en el precio de los inmuebles y de $0,6 \%$ en el caso de Santander. En el caso de Roma el indicador gravitatorio de accesibilidad también resultó ser significativo con un aumento en el precio medio de los inmuebles de 3,9\% por unidad adicional.

\begin{tabular}{ccccc}
\hline Variable & $\mathbf{R - 3}$ & $\mathbf{R}-\mathbf{4}$ & $\mathbf{S - 3}$ & $\mathbf{S}-\mathbf{4}$ \\
& & & & \\
\hline POP & $-0,000$ & $-0,000$ & $-0,000$ & $-0,000$ \\
& $(, 078)$ & $(, 059)$ & $(, 160)$ & $(, 016)$ \\
EMP & 0,000 & 0,000 & 0,000 & 0,000 \\
& $(, 226)$ & $(, 038)$ & $(, 502)$ & $(, 227)$ \\
ACCA & 0,039 & - & 0,037 & - \\
& $(, 000)$ & & $(, 428)$ & \\
CBD & - & $-0,007$ & - & $-0,006$ \\
& & $(, 000)$ & & $(, 004)$ \\
TRANS & $-0,084$ & $-0,007$ & 0,417 & 0,195 \\
& $(, 643)$ & $(, 993)$ & $(, 000)$ & $(, 160)$ \\
CEN & 0,127 & 0,195 & $-0,368$ & $-0,524$ \\
& $(, 262)$ & $(, 055)$ & $(, 110)$ & $(, 041)$ \\
COM & $-0,075$ & $-0,129$ & 0,020 & 0,080 \\
& $(, 615)$ & $(, 186)$ & $(, 704)$ & $(, 406)$ \\
GREEN & $-0,171$ & $-0,045$ & 0,350 & 0,333 \\
& $(, 060)$ & $(, 594)$ & $(, 004)$ & $(, 000)$ \\
PG & 0,521 & 0,456 & 0,372 & 0,304 \\
& $(, 000)$ & $(, 000)$ & $(, 021)$ & $(, 035)$ \\
\hline
\end{tabular}

Tabla 6- Parámetros estimados con los impactos totales de los modelos SAR

\section{DISCUSIÓN Y CONCLUSIONES}

Este artículo ha presentado la estimación de modelos de regresión hedónica y de modelos espaciales de regresión hedónica, para determinar la existencia y la magnitud de los impactos de la accesibilidad a oportunidades mediante transporte público en los valores inmobiliarios de las ciudades de Roma y Santander.

Los resultados confirman que la accesibilidad fue un factor significativo en el incremento del precio de los valores inmobiliarios en ambas áreas de estudio. Sin embargo, mientras que en el caso de Roma tanto el indicador gravitatorio como el indicador de accesibilidad relativa al centro urbano captaron este efecto, en el caso de Santander únicamente el tiempo de viaje al centro urbano fue una variable significativa. Esto seguramente se debió al carácter más monocéntrico del área urbana de Santander respecto del de Roma cuya zona de influencia presenta dos subcentros importantes además del centro histórico tradicional. La cobertura de las paradas de transporte público no mostró ser una variable relevante en el caso de Roma, mientras que en Santander, en los modelos donde se especificó junto con la variable CBD, no fue una variable claramente significativa.

En cuento a la correlación espacial en los residuos, en Roma ésta fue claramente significativa y fuerte, mientras que en el área de estudio de Santander fue débil y los modelos SAR no 
mejoraron significativamente la bondad de ajuste respecto a los modelos hedónicos convencionales. Por lo tanto, el mejor ajuste de los modelos espaciales en los datos de Roma se debió a la existencia de efectos de difusión e influencia entre zonas en los precios inmobiliarios, un fenómeno que no estuvo presente en Santander quizás porque los efectos de difusión quedaron neutralizados por la agregación zonal. Finalmente el cálculo de los impactos totales en los modelos SAR permitió comprobar cómo los efectos de la accesibilidad eran superiores a los estimados en los modelos de regresión hedónica no espaciales y superiores en el caso de Roma al caso de Santander, algo de acuerdo a las expectativas dado los mayores problemas de movilidad presentes en Roma.

La existencia de estos efectos de capitalización de la accesibilidad en los valores inmobiliarios avala la posibilidad de utilizar políticas de captura del valor en las áreas estudiadas de cara a mejorar la financiación del transporte público. Unas políticas que ya se han implementado de forma exitosa en otras áreas urbanas.

\section{ACKNOWLEDGEMENTS}

Esta investigación ha sido posible gracias a la financiación obtenida del Proyecto PARKINFO (TRA2013-48116-R) financiado por el Ministerio de Economía y Competitividad del Gobierno de España.

\section{REFERENCES}

Anselin, L. (1988) Spatial econometrics : methods and models. Kluwer Academic Publishers, Dordrecht; Boston.

Arnott, R.J., Stiglitz, J.E. (1979) Aggregate land rents, expenditure on public goods, and optimal city size. The Quarterly Journal of Economics, 471-500.

Bitter, C., Mulligan, G., Dall'erba, S. (2007) Incorporating spatial variation in housing attribute prices: a comparison of geographically weighted regression and the spatial expansion method. J Geograph Syst 9, 7-27.

Bowes, D.R., Ihlanfeldt, K.R. (2001) Identifying the Impacts of Rail Transit Stations on Residential Property Values. Journal of Urban Economics 50, 1-25.

Boyle, M., Kiel, K. (2001) A Survey of House Price Hedonic Studies of the Impact of Environmental Externalities. Journal of Real Estate Literature 9, 117-144.

Cascetta, E. (2009) Transportation systems analysis : models and applications, 2nd ed. Springer, New York.

Cervero, R., Kang, C.D. (2011) Bus rapid transit impacts on land uses and land values in Seoul, Korea. Transport Policy 18, 102-116.

Coppola, P., Nuzzolo, A. (2011) Changing accessibility, dwelling price and the spatial distribution of socio-economic activities. Research in Transportation Economics 31, 6371.

Court, A.T. (1939) Hedonic Price Indexes With Automobile Examples. The dynamics of automobile demand. General Motors, New York.

Debrezion, G., Pels, E., Rietveld, P. (2007) The Impact of Railway Stations on Residential 
and Commercial Property Value: A Meta-analysis. The Journal of Real Estate Finance and Economics 35, 161-180.

Dubé, J., Thériault, M., Des Rosiers, F. (2013) Commuter rail accessibility and house values: The case of the Montreal South Shore, Canada, 1992-2009. Transportation Research Part A: Policy and Practice 54, 49-66.

Fogarty, N.A., America, R. (2008) Capturing the value of transit. Center for TransitOriented Development.

Halvorsen, R., Palmquist, R. (1980) The Interpretation of Dummy Variables in Semilogarithmic Equations. American Economic Review 70, 474-475.

Handy, S.L., Niemeier, D.A. (1997) Measuring accessibility: An exploration of issues and alternatives. Environment and Planning A 29, 1175-1194.

Horner, M.W. (2004) Spatial Dimensions of Urban Commuting: A Review of Major Issues and Their Implications for Future Geographic Research. The Professional Geographer $56,160-173$.

Ibeas, A., Cordera, R., dell'Olio, L., Coppola, P., Dominguez, A. (2012) Modelling transport and real-estate values interactions in urban systems. Journal of Transport Geography 24, 370-382.

Koenig, J.G. (1980) Indicators of urban accessibility: Theory and application. Transportation 9, 145-172.

LeSage, J.P., Pace, R.K. (2009) Introduction to spatial econometrics. CRC Press, Boca Raton.

Lesage, J.P., Pace, R.K. (2010a) The Biggest Myth in Spatial Econometrics. Available at SSRN: http://ssrn.com/abstract=1725503 or http://dx.doi.org/10.2139/ssrn.1725503.

LeSage, J.P., Pace, R.K. (2010b) Spatial Econometric Models. Handbook of Applied Spatial Analysis: Software Tools, Methods and Applications. Springer, Berlin, pp. 355-376.

Malpezzi, S. (2008) Hedonic Pricing Models: A Selective and Applied Review. Housing Economics and Public Policy ed Tony O'Sullivan, K.G., pp. 67-89.

Martínez, F.J. (2000) Towards a Land-use and transport interaction framework. Handbook of Transport Modelling eds Hensher, D.A., Button, K.J. Elsevier Science, pp. 145-164.

Munoz-Raskin, R. (2010) Walking accessibility to bus rapid transit: Does it affect property values? The case of Bogotá, Colombia. Transport Policy 17, 72-84.

Rosen, S. (1974) Hedonic Prices and Implicit Markets: Product Differentiation in Pure Competition. Journal of Political Economy 82, 34-55.

Senior, M.L. (2009) Impacts on travel behaviour of Greater Manchester's light rail investment (Metrolink Phase 1): evidence from household surveys and Census data. Journal of Transport Geography 17, 187-197.

Wang, Y., Kockelman, K.M., Wang, X. (2013) Understanding spatial filtering for analysis of land use-transport data. Journal of Transport Geography 31, 123-131. 